\title{
Effects of coconut water on blood sugar and retina of rats with diabetes
}

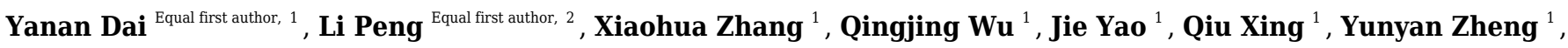 \\ Xiaobo Huang ${ }^{1}$, Shaomei Chen ${ }^{1}$, Qing Xie ${ }^{\text {Corresp. } 1}$ \\ ${ }^{1}$ Department of Ophthalmology, Central South University Xiangya School of Medicine Affiliated Haikou Hospital, Haikou , Hainan,P.R. China, Haikou, China \\ 2 Department of Ophthalmology, The Second Xiangya Hospital $\square$ Central South University,Changsha,Hunan,P.R. China, Changsha, China \\ Corresponding Author: Qing Xie \\ Email address: opht_xq@163.com
}

Background: This study aimed to evaluate the effects of coconut water on the general condition(Fasting blood sugar and body weight) and retina of diabetic rats. Methods: Fortyeight Sprague-Dawley male rats were divided into normal controls (NC), diabetes mellitus (DM), diabetes+coconut water (DM+CW), and diabetes+glibenclamide (DM+Gli) groups. After 4 weeks of normal feeding, coconut water was given to group III-DM+CW and 0.6 $\mathrm{mg} / \mathrm{kg}$ glibenclamide to group IV-DM+Gli. The blood sugar, body weight, total retinal thickness, pathological changes, and VEGF expression in the retina were analyzed at different time points. Results: The fasting blood sugar was 4-6 $\mathrm{mmol} / \mathrm{L}$ in group I-NC and continuously increased in group II-DM, whereas gradually decreased after the 4th experiment week in the remaining two groups. The rats, except in group I-NC, have lost weight. In group II-DM, the total retinal thickness was significantly increased after the 8th and 12th experiment week, and the pathological changes in retina were observed. VEGF was almost fully expressed in the ganglion cell layer and inner granular layer and partially expressed in the outer granular layer in group II-DM, and mainly expressed in the ganglion cell layer and inner layer in group I-NC, with a lighter color. Group III-DM + CW and group IV-DM + Gli demonstrated similar VEGF expression as in group I-NC. Conclusions $\square$ Coconut water has the potential to reduce blood sugar and diabetic retinal damage, serving as a candidate drug or nutrient for treating diabetes and its complications. 
1 Effects of coconut water on blood glucose and retina of diabetic rats

2 Authors: Yanan Dai1†, Li Peng2†, Xiaohua Zhang1, Qingjing Wu1, Jie Yao1, Qiu Xing1,

3 Yunyan Zheng1, Xiaobo Huang1, Shaomei Chen1, Qing Xie1*

41 Department of Ophthalmology, Central South University Xiangya School of Medicine

5 Affiliated Haikou Hospital, Haikou, Hainan, P.R. China

62 Department of Ophthalmology, The Second Xiangya Hospital, Central South

7 University,Changsha, Hunan,P.R. China

$8 \uparrow$ These authors contributed equally to this work.

9 Corresponding author: Qing Xie

10 RenMin Rd. \#43, Meilan District, Haikou, Hainan, 570208, P. R. China.

11 E-mail: opht_xq@163.com

12 Tel: +86-13118989700

13 Fax: +86-0898-66189688

\section{Abstract}

20 Background: We evaluated the effects of coconut water on the fasting blood glucose, body

21 weight, and retina of diabetic rats.

22 Methods: Forty-eight Sprague-Dawley male rats were divided into normal controls (I-NC),

23 diabetes mellitus (II-DM), diabetes + coconut water (III-DM+CW), and diabetes + glibenclamide

24 (IV-DM+Gli) groups. After 4 weeks of normal feeding, coconut water was given to the III- 
$25 \mathrm{DM}+\mathrm{CW}$ group and $0.6 \mathrm{mg} / \mathrm{kg}$ glibenclamide was given to the IV-DM+Gli group. We analyzed

the blood glucose, body weight, total retinal thickness, pathological changes, and vascular endothelial growth factor(VEGF) expression in the retina of each rat at different time points. Results: The fasting blood glucose of rats in the I-NC group averaged 4-6 mmol/L, with an increase in the II-DM group. Blood glucose levels gradually decreased after the 4th experimental week in the remaining two groups. The rats exhibited weight loss in all groups, except in group INC. In group II-DM, the total retinal thickness increased significantly after the 8th and 12th experimental week, and pathological changes in the retina were observed. The II-DM group also showed an almost full expression of VEGF in the ganglion cell layer and inner granular layer, and partial expression. VEGF in group I-NC was mainly expressed in the ganglion cell layer and inner layer, with a lighter color. Group III-DM + CW and group IV-DM + Gli demonstrated VEGF expression similar to that of group I-NC.

Conclusions: Coconut water may reduce blood glucose and diabetic retinal damage and may be a candidate drug or nutrient for treating diabetes and its complications.

\section{Introduction}

Diabetes is one of the most significant public health problems worldwide. According to the International Diabetes Federation, there were 382 million diabetics worldwide in 2013 with a projected 592 million in 2035 (IDF Diabetes Atlas, 6th ed.). Diabetes mellitus (DM) causes acute metabolic disorder syndrome, leading to long-term damage, dysfunction, and failure of some organs due to chronic hyperglycemia, and may lead to death. Among these complications, diabetic retinopathy (DR) is one of the predominant microvascular complications of type 1 or type $2 \mathrm{DM}$ and is a major ocular complication. Developed countries, including Europe and America, have conducted a number of epidemiological studies on diabetic retinopathy. According to the Eye Diseases Prevalence Research Group, an American research organization, the incidence of diabetic retinopathy can be as high as 40.3\% (Kempen et al., 2004). According to the National Health and Nutrition Examination Survey, the incidence rate of diabetic retinopathy in patients over 40 years old was $28.5 \%$ (2010). The incidence of DR in Chinese patients with DM is approximately 3.3\%-7.4\%. The visual impact of proliferative retinopathy has 
54 serious, potentially negative implications for the employment and life of diabetic patients (Gu et 55 al., 2020). acetylglucosamine, and the activation of protein kinase $\mathrm{C}(\mathrm{PKC})$ leading to the formation of the advanced glycation end product. Disorderly regulation of these biochemical mechanisms at the molecular level is the main pathogenesis of DR. The current clinical treatments tend to focus on diabetic macular edema and proliferative diabetic retinopathy. These conditions are treated with intravitreal applications of various VEGF antagonists, corticosteroids, and vitreous surgery. The results of these treatments are unsatisfactory and expensive and they do not prevent or reverse the progression of diabetic retinopathy.

Early intervention is important to prevent or delay the development of DR, and to prevent and treat blindness secondary to this disease. Effective drug treatments for early diabetic retinopathy are still being explored.

Coconut water $(\mathrm{CW})$ is a natural beverage obtained from the cavity of the coconut fruit. Recent animal experiments have reported that $\mathrm{CW}$ can reduce blood glucose levels, regulate carbohydrate metabolism, and improve antioxidant capacity. Several studies have reported that CW may alleviate kidney damage caused by diabetes (Nwangwa 2012; Pinto et al. 2015) \{Nwangwa, 2012 \#5;Pinto, 2015 \#6;Prathapan, 2011 \#7\}(Prathapan \& Rajamohan, 2011; Preetha et al., 2012; Preetha et al., 2013). Both DR and diabetic nephropathy are retinal microangiopathic diseases and it is unknown whether $\mathrm{CW}$ can alleviate diabetic retinal damage.

Glibenclamide is a hypoglycemic medication that stimulates pancreatic beta cells. Its availability and affordability makes glibenclamide popular for the management of type 2 diabetes mellitus (Rambiritch et al., 2007). We sought to evaluate the effects of CW on the fasting blood glucose, 
81 body weight, and retinal protection of diabetic rats. We also explored the usefulness of natural

82 drugs in the treatment of DR.

84 Material and methods

85 Animals

86 We studied 48 10-week-old male Sprague-Dawley (SD) rats, weighing 350 $\pm 30 \mathrm{~g}$ with clear ocular

87 media and no ocular fundal lesions when examined by slit lamp microscope and ophthalmoscope.

88 The rats were kept in a pathogen-free facility with access to food and water under controlled conditions (temperature $18-26^{\circ} \mathrm{C}$; humidity $40-70 \%$; and $12-\mathrm{h}$ light/dark cycle). All SD rats and standard feed were purchased from Changsha Tianqin Biotechnology Co., Ltd. (SCXK, Xiang, 2014-0011). All SD rats and their feeds were tested physically and chemically by the Center for Disease Control and Prevention of Hunan Province (sample acceptance Nos. 2017DW041, No.2017DS009). All experimental procedures were approved by the biomedical ethics committee of Haikou People's Hospital. (2017-087), and all efforts were made to minimize the suffering of the test subjects.

Local mature coconuts (6-8 months old) were purchased weekly in Wenchang, Hainan to ensure the consistency of the $\mathrm{CW}$. The $\mathrm{CW}$ was collected and stored in a refrigerator at $4{ }^{\circ} \mathrm{C}$ for experimentation. We obtained the following materials for use in our study: Glibenclamide (H12020790, Tianjin Pacific Pharmaceutical Co., Ltd.); Streptozotocin (S0130, Beijing Sein Tan Technology Co., Ltd); citric acid-sodium citrate buffer (pH 4.5) (Xiamen Sea Standard Technology Co., Ltd); ketamine (Daiichi-Sankyo, Co., Ltd., Tokyo, Japan); xylazine (Bayer AG, Leverkusen, Germany); rabbit anti-mouse VEGF antibody (p80084, PhD Bio Company); fast enzyme-labeled sheep anti-mouse/rabbit IgG polymer (kit-5930, Fuzhou Maixin Biotechnology Development Co., Ltd.); slit lamp microscope (SL-3G, Topcon); ophthalmology (YZ11D, Suzhou Liuliu Vision Technology Co., Ltd.); FreeStyle Optium H (Abbott Company, USA); Optical coherence tomography [(SPECT) RALIS OCT, Heidelberg, Germany]; electronic balance (XPE105, METTLER TOLED O, Switzerland); and a full automatic HE dyeing machine (Coverstainer, Dako, Denmark). 
110 Diabetes model

111 All male SD rats were fasted for 12 hours but had free access to water. Twelve rats were

112 randomly selected for group I-NC using the random number table method. Sodium citrate buffer

113 ( $\mathrm{pH} 4.5$ ) was injected intraperitoneally at a dose of $60 \mathrm{mg} / \mathrm{kg}$ body weight. We induced diabetes

114 mellitus in the remaining 36 rats using an intraperitoneal injection of 1\% streptozotocin (STZ)

115 solution dissolved in citric acid-sodium citrate buffer $(\mathrm{pH} 4.5)$. The rats were fed 30 minutes after

116 the injection. Tail blood was then drawn to measure their blood glucose levels and rats with blood

117 glucose less than $16.7 \mathrm{mmol} / \mathrm{L}$ were excluded.

119 Experimental grouping

120 Our diabetic model was successfully established and the experimental period lasted for 20 weeks.

121 Twelve normal rats were included in group I-NC. Thirty-six diabetic rats were randomly divided

122 into the II-DM group, III-DM + CW group, and IV-DM + Gli group, with 12 rats in each group.

123 All groups were acclimatized for 1 month with normal rodent chow diet and water ad libitum

124 prior to the start of the study. After 1 month, all groups were treated as follows for the remainder

125 of the 20 weeks:

126 Group I-NC: $\mathrm{n}=12$; Normal rodent chow diet and water ad libitum

127 Group II-DM: $\mathrm{n}=12$; Normal rodent chow diet and water ad libitum

128 Group III-DM + CW: $\mathrm{n}=12$; Normal rodent chow diet and CW ad libitum

129 Group IV-DM + Gli: n=11; Normal rodent chow diet and water ad libitum + daily oral Gli at

130 dose of $0.6 \mathrm{mg} / \mathrm{kg}$ (Preetha et al., 2013).

131

132 Experimental detective index

133 General state

134

135 We restricted access to food and water for 8 hours every 4 weeks and then weighed the rats and

136 drew tail blood samples to measure the fasting blood glucose from each subject.

137

138 Imaging examination: Optical coherence tomography (OCT) 
139 We examined the left eye of each rat, in each group, by OCT in the 8th, 12th, 16th, and 20th

140 experimental weeks. The rats were weighed and then injected intraperitoneally with a mixture of

141 ketamine $(80 \mathrm{mg} / \mathrm{kg})$ and xylazine $(10 \mathrm{mg} / \mathrm{kg})$. The left eye was dilated using topicamide eye

142 drops and the corneal surface was treated with sodium hyaluronate eye drops to keep the cornea

143 moist. The entire body of the test subject was wrapped with a sterile cloth after being placed in an

144 anesthesia-induced coma. The subject was laid on the OCT examination table so that both eyes

145 could be examined to evaluate if the anterior and posterior diameters of the eyes were consistent

146 with the scanning light source. The OCT examination was conducted by a second researcher and

147 all examinations were performed by the same deputy chief physician.

148 We manually measured the distance from the inner limiting membrane to the retinal pigment

149 epithelium (the thickness of the retina) using the OCT detection system. The central part of the

150 posterior pole of the retina in rats involves the optic disc, so the total retinal thickness was

151 measured at the disc diameter (DD) of the upper, lower, nasal, and temporal discs of the optic

152 disc to facilitate an accurate measurement. The mean value of the 4 data points was taken as the

153 total retinal thickness. Diabetes mellitus in the subjects led to cataract formation, which affected

154 the signal strength of the OCT. The examination in all groups was terminated when half the rats

155 in a particular group were unable to have a sufficient signal strength to perform OCT, to allow for

156 comparison between groups.

\section{Tissue processing/photomicrography}

159 The rats were anesthetized as mentioned above prior to sacrifice by cervical dislocation 1-7 days

160 after laser-treatment, at the end of the experimental period in the $20^{\text {th }}$ week. The left eyeball was

161 removed and quickly placed in an eyeball fixative solution (formaldehyde stock solution:glacial

162 acetic acid:95\% ethanol:distilled water $=2: 1: 10: 7)$ for $24 \mathrm{~h}$, samples were then embedded in

163 paraffin and cut into 4- $\mu \mathrm{m}$-thick continuous sheets.

164 HE staining: Paraffin specimens were dewaxed with xylene, hydrated with gradient ethanol,

165 long-form hematoxylin and eosin (HE) stained, and ethanol dehydrated. Neutral gum sealing was 
166 performed after the xylene was transparent. An optical microscope was used to observe the

167 thickness of the retina and the shape and number of cells in each layer.

168 VEGF immunohistochemical staining: We performed a conventional dewaxing of the sample and

169 then immersed the sample in citric acid buffer solution $(\mathrm{pH}=6.0)$. We used the heat-induced

170 antigen repair method with a microwave oven after selection. Normal goat serum was used as a

171 blocking solution, and the rabbit anti-mouse VEGF antibody $(1: 100)$ was added at $4^{\circ} \mathrm{C}$ and left

172 overnight. After rewarming, the enzyme-labeled goat anti-mouse/rabbit IgG polymer was added

173 dropwise and incubated at room temperature for $15 \mathrm{~min}$. The specimen was washed and then

174 incubated in the working solution for $15 \mathrm{~min}$ at room temperature. The sheet was counterstained,

175 dehydrated, cleared, and sealed after DAB staining.

176 The expression of VEGF-positive cells in the retina of each group of rats was observed under

177 light microscope. The positive expression of VEGF in the retina was yellow-brown in color.

179 Statistical Analysis

180 The experimental data were analyzed and compared using SPSS 20.0 statistical software (IBM

181 Corp), and the experimental data of each group were expressed by means $\square$ SD. A single sample

182 K-S test with a nonparametric test was used to verify the normality of the data. A single factor

183 analysis of variance was used for multigroup statistical method to measure the normally

184 distributed data. An LSD-t test was used for two-way comparison between groups. The Kruskal-

185 Wallis $\mathrm{H}$ test was used for multigroup analysis for measuring the data of normal distribution, and

186 the Mann-Whitney U test was used for intergroup analysis. The test level was set at alpha=0.05.

187 The difference was statistically significant when the $\mathrm{p}$ values were $<0.05$.

189 Results

190 Diabetes model

191 Two rats were excluded because of hyperglycemia. The success rate of the established DM model

192 was $94.44 \%$ and included rats whose blood glucose levels did not reach the standard and died

193 within three days after STZ injection. Thirty-four rats successfully established DM. To ensure the

194 same number of rats in each group, one rat was randomly excluded by number table. The 
195 remaining rats were divided into three groups: group II-DM, group III-DM $+\mathrm{CW}$, and group IV-

$196 \mathrm{DM}+\mathrm{Gli}$. One rat from group I-NC was excluded using the same method, and 11 rats were

197 guaranteed in each group.

198

199 General state

200 Fasting blood glucose

201 The fasting blood glucose of rats in group I-NC was always at a normal level (4-6 mmol/L)

202 during the experimental process and the remaining three groups had significantly higher levels.

203 The difference was statistically significant $(\mathrm{P}>0.05)$. The fasting blood glucose of group II-DM

204 gradually increased during the $4^{\text {th }}$ experimental week and maintained high levels, reaching 31.41

$205( \pm 2.64 \mathrm{mmol} / \mathrm{L})$ by the $20^{\text {th }}$ experimental week.

206 However, the blood glucose levels of groups III-DM+CW and IV-DM+Gli gradually decreased.

207 The blood glucose levels of groups III-DM+CW and IV-DM+Gli decreased to $24.01 \pm 1.05 \mathrm{mmol} /$

$208 \mathrm{~L}$ and $24.25 \pm 1.15 \mathrm{mmol} / \mathrm{L}$, respectively, in the $20^{\text {th }}$ experimental week. The values were far

209 higher than group I-NC, but were lower than group II-DM, and the difference was statistically

210 significant $(\mathrm{P}>0.05)$, (Figure 1).

\section{Body weight}

213 There was no significant difference in the initial body weight of rats among the four groups (P >

214 0.05). During the experimental period, the rats in group I-NC showed a steady increase in weight

215 when compared to the other three groups, while the weight of the rats in the remaining three

216 groups decreased by varying degrees, showing no significant difference among the three groups

217 (P $>0.05)$. In the $4^{\text {th }}$ experimental week, the drinking water of group III-DM+CW was replaced

218 with CW. Glibenclamide was administered until the $20^{\text {th }}$ experiment week in group IV-DM+Gli,

219 and there was still no significant difference in the body weight among the three groups $(\mathrm{P}>0.05)$

220 (Figure 2). 
223 More than half of the rats in the II-DM group developed severe cataracts in the $16^{\text {th }}$ experimental

224 week, so the OCT signal strength was insufficient for examination. The examination was

225 terminated in all groups. Therefore, only the results of experimental weeks 8 and 12 were

226 obtained. The SD rat's retina does not have a macula, but its layers have the same structure as a

227 human's and OCT allows for the assessment of individual layers of the retina. (Figure 3). The

228 results in the $8^{\text {th }}$ experimental week revealed that the mean of total retinal thickness of group II-

229 DM was significantly increased, and the difference was statistically significant when compared

230 with the other three groups $(\mathrm{P} 1=0.001, \mathrm{P} 2=0.002, \mathrm{P} 3=0.000<0.05$, respectively). No significant

231 difference was observed among the other three groups $(\mathrm{P}=0.201>0.05)$.

232 The total retinal thickness of group II-DM was the highest among the three groups over time, 233 showing a significant difference among the other three groups $(\mathrm{P} 1=0.002, \mathrm{P} 2=0.001, \mathrm{P} 3=0.006<$

234 0.05). No significant difference was observed among the other three groups at the $12^{\text {th }}$

235 experimental week $(\mathrm{P}=0.824>0.05$, Table 1 , Figure 4$)$.

\section{3.4 HE staining image of rat retinas}

238 The retinal nerve fiber layer and the outer subordinate layer remained very thin in group I-NC.

239 The inner plexiform layer was the thickest, the inner granular layer and the outer granular layer

240 cells were arranged neatly and connected tightly, and the structure of each layer of the retina was

241 clear with no abnormalities.

242 However, in group II-DM, the inner and outer granular layers of the retina were slightly thinner,

243 the number of cells was decreased, the arrangement was disordered, and no other obvious

244 abnormalities were observed. The granular layer thickness of groups III-DM+CW, IV-DM+Gli

245 and group I-NC was similar and was arranged in a disordered manner with no obvious

246 abnormalities (Figure 5).

\subsection{VEGF immunohistochemical staining of retinal sections of rats}

249 In the rat retina, VEGF protein positive expression is shown by a yellow-brown color and was

250 mostly expressed in the cytoplasm and membrane. In group II-DM, VEGF was almost fully 
251 expressed in the ganglion cell layer and inner granular layer, and partially expressed in the outer

252 granular layer. In group I-NC, VEGF was mainly expressed in the ganglion cell layer and the

253 inner layer, and the color was lighter. Group III-DM + CW and group IV-DM + Gli demonstrated

254 similar VEGF expression as in group I-NC, appearing mainly in the ganglion cell layer and inner

255 plexiform layer (Figure 6).

257 Discussion

258 Our study showed that CW may have the potential to reduce blood glucose and diabetic retinal 259 damage, but has no effect on weight change caused by DM. Coconut is a very common tropical 260 plant. CW can be used as a potential candidate nutrient for treating diabetes and its 261 complications, and has broad prospective applications.

262 In this study, insulin-dependent diabetes was successfully induced in rats by injecting STZ into 263 the abdominal cavity to destroy the pancreatic islet beta cells. Typical diabetic changes such as 264 elevated blood glucose and weight loss occurred during the experimental cycle. CW did not 265 promote weight loss in rats but it partially reduced the blood glucose. Blood glucose was not 266 reduced to normal in this study group, but it was significantly lower than that in the diabetic 267 group. The hypoglycemic effect was similar to that of glibenclamide, which is the standard drug 268 for DM. Our results were similar to those of Preetha et al. (2013b). Previous animal experiments 269 have found that CW can reduce blood glucose in alloxan-induced diabetic rats (Pinto et al., 270 2015). During pathological examination, CW was found to reduce pancreatic injury and stimulate 271 islet beta cell regeneration in diabetic rats (Preetha et al., 2013). These results suggest that CW 272 can effectively reduce blood glucose in diabetic rats, but its mechanism remains unknown and 273 requires further experimental studies.

274 The pathophysiological mechanism of DR is very complex. In the early stages, the inflammatory 275 damage of endothelial cells, apoptosis, and the shedding of pericytes and endothelial cells is 276 caused by leukocyte adhesion. The reduced endothelial tight junction protein and thickened 277 basement membrane causes damage to the inner blood-retinal barrier. This causes a disturbance 278 in the glial inner layer of the retinal nerve and the functionality of the blood vessels, increasing 279 the permeability of the blood vessels, and leakage of the retinal tissue (Chibber et al., 2007; 
280 Joussen et al., 2001; Nag et al., 2011; Tang \& Kern, 2011; van Hecke et al., 2005). OCT may

281 detect the changes in the retinal thickness caused by retinal tissue leakage at an early stage and

282 assist in providing quantitative information. HE staining on the retinal section of rats revealed the 283 extent of damage to the layers of the retina at the cellular level. In this experiment, the increased 284 thickness of the retina was observed in the 8th and $12^{\text {th }}$ experimental week in rats with diabetes, 285 suggesting that retinal tissue leakage occurred in the early stages.

286 We were unable to obtain data for the next few weeks of the experiment. The retinal thickness in 287 groups II-DM + CW and IV-DM + Gli was the same as that in normal rats. HE staining of the 288 retinal sections revealed that in group II-DM, visible pathological changes were noticed in the 289 retina at the $20^{\text {th }}$ week, such as thinner inner and outer granular layers reduced the number of cells 290 and disordered arrangement.

291 However, these changes were not observed in the retinal layers of group II-DM+CW. These 292 results indicated that $\mathrm{CW}$ may reduce cellular damage in various layers of the retina and reduce 293 leakage of retinal tissues.

294 VEGF is currently the most important and directly stimulating angiogenic factor. It has strong 295 vascular permeability, can significantly increase microvascular permeability, and destroy BRB 296 (Praidou et al., 2010). Various angiogenesis-related factors promote the proliferation and permeability of microvascular endothelial cells by directly or indirectly inducing the expression of VEGF and its receptors (Rakhila et al., 2016). VEGF is closely related to retinal tissue 299 leakage.

300 VEGF immunohistochemical observation was performed on the retina of rats. In the $20^{\text {th }}$ experimental week, VEGF was almost fully expressed in the ganglion cell layer, inner plexiform

302 layer, and granular layer in the II-DM group, and was partially expressed in the outer granular 303 layer. The retinal VEGF was mainly expressed in the ganglion cell layer and the inner layer in 304 group III-DM + CW with a lighter color; the appearance was similar to that found in group I-NC. 305 We should seek to find how CW can reduce the retinal VEGF expression level, affecting retinal 306 vascular permeability and reducing retinal tissue leakage.

307 Diabetes may cause hyperglycemic episodes which in turn impacts five key biochemical 308 pathways: the polyol pathway activation; the production of advanced glycation end products 
309 (AGEs); protein kinase C (PKC) activation; hexosamine pathway activation; and poly (ADP-

310 ribose) polymerase upregulation. Several DR mechanisms have been shown to be involved in the

311 overexpression of VEGF. Activated protein kinase C (PKC) can promote an increase in VEGF

312 expression levels (Amadio et al., 2012). The upregulation of the expression of VEGF is affected

313 by the formation of advanced glycation end-products (AGEs) and the activation of the receptor

314 for advanced glycation end-products (Kandarakis et al., 2014; Yan et al., 2010). The five key

315 biochemical pathways lead to oxidative stresses, resulting in mitochondrial dysfunction,

316 deregulation of proinflammatory mediators, and hypoxia. These effects cause the apoptosis of

317 vascular and neuronal cells and the upregulation of VEGF expression. The generation of ROS

318 and oxidative stress further exacerbates metabolic dysfunction, leading to elevated ROS

319 production in a self-perpetuating positive feedback mechanism (Wang et al., 2017).

320 This reveals the important role of antioxidants in DR. CW is a natural beverage obtained directly

321 from the cavity of the coconut fruit. It has a very high nutritional value and acts as a natural

322 antioxidant. Clinical trials revealed that CW may increase antioxidant enzymes and reduce lipid

323 peroxidation (Santosa, 2015). Many oxygen free radical scavengers, such as ascorbic acid,

324 cysteine, phenolic compounds, and L-arginine are present in CW and protect organs via their

325 antioxidant capabilities (Amadio et al., 2012; Rakhila et al., 2016). CW may act to remove ROS

326 and reduce OS, reduce the activation of PKC and formation of AGEs, decrease the expression of

327 VEGF, and decrease the leakage of retinal tissue through its antioxidant properties. However, the

328 specific mechanisms of action require further exploration.

330 Conclusions

$331 \mathrm{CW}$ has the potential to reduce blood glucose and diabetic retinal damage but it has no effect on

332 weight change caused by DM. We speculated that this may be due to its antioxidant properties.

333 Further studies are needed to investigate the role of coconut water supplementation in DM and

334 which antioxidants in coconut water may be used to develop candidate drugs.

335

336 Acknowledgments 
337 The authors appreciate the support of the Central Laboratory and Pathology, Department of

338 Haikou Hospital affiliated with Xiangya Medical College of Central South University.

\section{References}

2010. Prevalence of Diabetic Retinopathy in the United States, 2005-2008. \%J Jama Journal of the American Medical Association.

Chibber R, Ben-Mahmud BM, Chibber S, and Kohner EM. 2007. Leukocytes in diabetic retinopathy. Curr Diabetes Rev 3:3-14. 10.2174/157339907779802139

Gu D, Agron S, May LN, Mirza RG, Telemedicine PJBJ, and e-Health. 2020. Nonmydriatic Retinal Diabetic Screening in the Primary Care Setting: Assessing Degree of Retinopathy and Incidence of Nondiabetic Ocular Diagnoses. 
366

367

368

369

370

371

372

373

374

375

376

377

378

379

380

381

382

383

384

385

386

387

388

389

390

Kempen JH, O'Colmain BJ, Leske MC, Haffner SM, and Ophthalmology RFHJAo. 2004. The prevalence of diabetic retinopathy among adults in the United States The Eye Diseases Prevalence Research Group Arch Ophthalmol 20041224552563 10.1001/archopht.122.4.552 15078674. 122:552-563.

Nwangwa EKJJoHS. 2012. The Reno-Protective Effects of Coconut Water on the Kidneys of Diabetic Wistar Rats.

Pinto IFD, Silva RP, Filho ADBC, Dantas LS, and Matos HRJJoMF. 2015. Study of Antiglycation, Hypoglycemic, and Nephroprotective Activities of the Green Dwarf Variety Coconut Water (Cocos nucifera L.) in Alloxan-Induced Diabetic Rats. 18:116125.

Praidou A, Androudi S, Brazitikos P, Karakiulakis G, Papakonstantinou E, and Dimitrakos SJCDR. 2010. Angiogenic growth factors and their inhibitors in diabetic retinopathy. 6:-.

Prathapan A, and Rajamohan TJJoFB. 2011. ANTIOXIDANT AND ANTITHROMBOTIC ACTIVITY OF TENDER COCONUT WATER IN EXPERIMENTAL MYOCARDIAL INFARCTION. 35:1501-1507.

Preetha PP, Devi VG, Rajamohan TJF, and Function. 2012. Hypoglycemic and antioxidant potential of coconut water in experimental diabetes. 3:753.

Preetha PP, Girija Devi V, and Rajamohan TJEJoIM. 2013. Effects of coconut water on carbohydrate metabolism and pancreatic pathology of alloxan induced diabetic rats. 5:234-240.

Rambiritch V, Pillai G, Maharaj B, and Robertson LIJSATVG. 2007. Glibenclamide--what dose? $97: 475$.

Wang JH, Ling D, Tu L, Van Wijngaarden P, Dusting GJ, Liu GSJP, and Therapeutics. 2017. Gene therapy for diabetic retinopathy: Are we ready to make the leap from bench to bedside? 173:1. 
391 Preetha PP, Girija DV, and Rajamohan T. 2013b. Comparative effects of mature coconut water 392 (Cocos nucifera) and glibenclamide on some biochemical parameters in alloxan induced diabetic rats. Revista Brasileira de Farmacognosia 23:481-487.

394

395

396

Joussen AM, Murata T, Tsujikawa A, Kirchhof B, Bursell SE, and Adamis AP. 2001. Leukocytemediated endothelial cell injury and death in the diabetic retina. Am J Pathol 158:147152. $10.1016 / \mathrm{s} 0002-9440(10) 63952-1$

Nag S, Kapadia A, and Stewart DJ. 2011. Review: molecular pathogenesis of blood-brain barrier breakdown in acute brain injury. Neuropathol Appl Neurobiol 37:3-23.10.1111/j.13652990.2010.01138.x

Tang J, and Kern TS. 2011. Inflammation in diabetic retinopathy. Prog Retin Eye Res 30:343-358.39710.1016/j.preteyeres.2011.05.002

van Hecke MV, Dekker JM, Nijpels G, Moll AC, Heine RJ, Bouter LM, Polak BC, and Stehouwer CD. 2005.Inflammation and endothelial dysfunction are associated with retinopathy: the Hoorn Study. Diabetologia 40048:1300-1306. 10.1007/s00125-005-1799$\mathrm{y}$

Rakhila H, Al-Akoum M, Doillon C, Lacroix-Pepin N, Leboeuf M, Lemyre M, Akoum A, and Pouliot M. 2016.Augmented Angiogenic Factors Expression via FP Signaling Pathways in Peritoneal Endometriosis. J Clin Endocrinol Metab 101:4752-4763. 10.1210/jc.20161569

Amadio M, Osera C, Lupo G, Motta C, Drago F, Govoni S, and Pascale A. 2012. Protein kinase $\mathrm{C}$ activation affects, via the mRNA-binding Hu-antigen R/ELAV protein, vascular endothelial growth factor expression in a pericytic/endothelial coculture model. Mol Vis $18: 2153-2164$.

Kandarakis SA, Piperi C, Topouzis F, and Papavassiliou AG. 2014. Emerging role of advanced glycation-end products(AGEs) in the pathobiology of eye diseases. Prog Retin Eye Res 42:85-102.36210.1016/j.preteyeres.2014.05.002 
417 Yan SF, Ramasamy R, and Schmidt AM. 2010. The RAGE axis: a fundamental mechanism 418 signaling danger to the vulnerable vasculature. Circ Res 106:842-853.

$419 \quad 10.1161 /$ circresaha.109.212217

420 Santosa STZAAS. 2015. Effects of Tender Coconut Water on Antioxidant Enzymatic Superoxida 421 Dismutase(SOD),CATALASE (CAT), Glutathione Peroxidase (GPx) and Lipid 422 Peroxidation In Mercury Exposure Workers.International Journal of Science and $423 \quad$ Research (IJSR) 12:518-519.

424 Amadio M, Osera C, Lupo G, Motta C, Drago F, Govoni S, and Pascale A. 2012. Protein kinase 425 C activation affects, via the mRNA-binding Hu-antigen R/ELAV protein, vascular 426 endothelial growth factor expression in a pericytic/endothelial coculture model. Mol Vis $427 \quad 18: 2153-2164$.

428 International Diabetes Fedaration.IDF Diabetes atlas.6th

429 editionn.[2014-06-30]http://www.idf.org/diabetesatlas

430 
Figure 1

Comparison of fasting blood sugar at different time points in rats of different groups

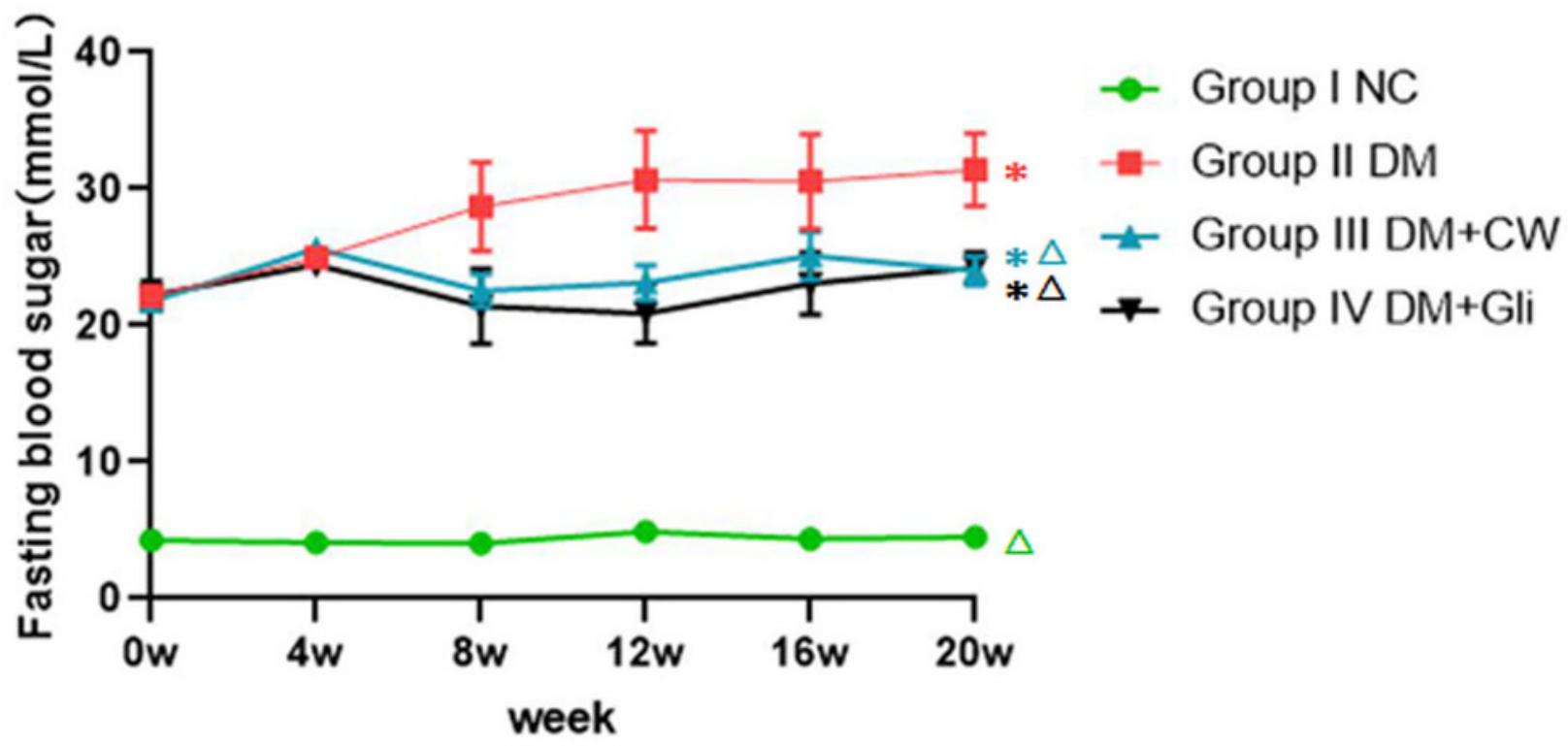

$* P<0.05$, Compared with Group I-NC

$\Delta P<0.05$, Comparison with Group II-DM 
Figure 2

Weight comparison of rats in each group at different time points

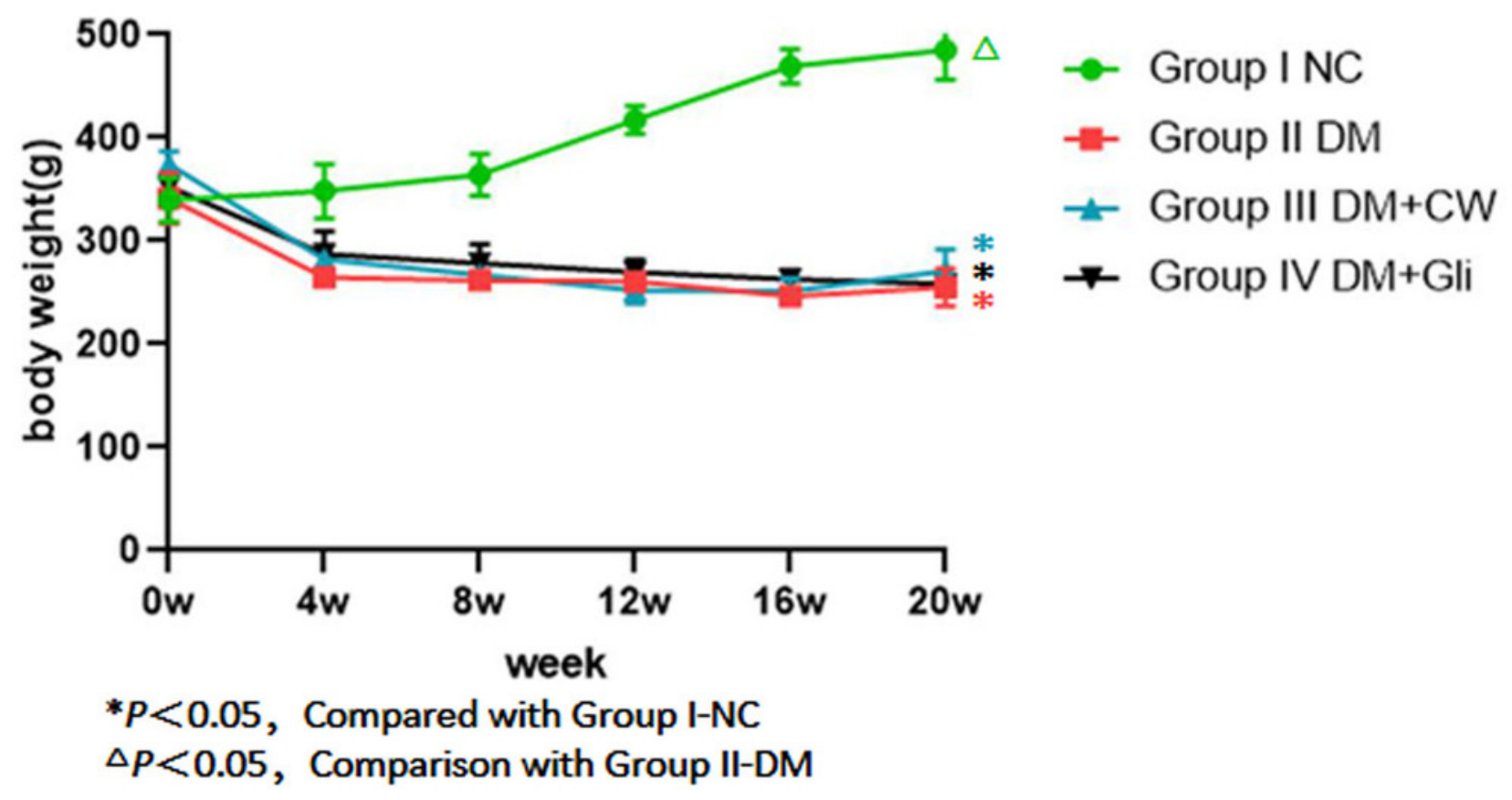




\section{Figure 3}

Corresponding relationship between OCT examination result and pathological section of rat retina (the range of blue arrow indicates total retinal thickness, $\mathrm{HE} * 400$ )

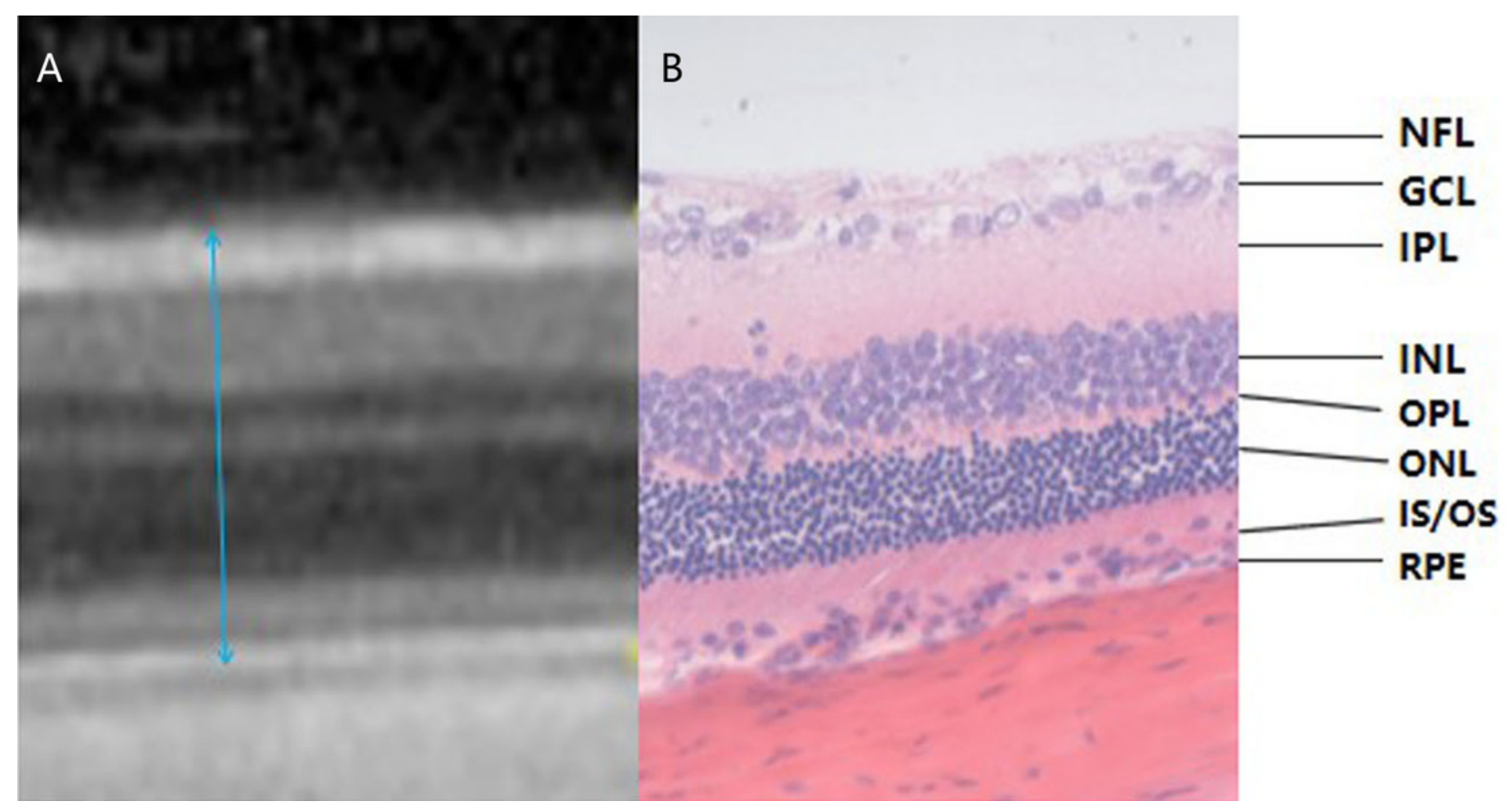




\section{Figure 4}

At the $12^{\text {th }}$ experiment week of each group, OCT was used to measure the 1DD images from the superior, inferior, nasal and temporal sides of the optic nerve.(A-D): Group INC, (E-H): Group II-DM, (I-L): Group III-DM+CW, (M-P): Group IV-DM+Gli.

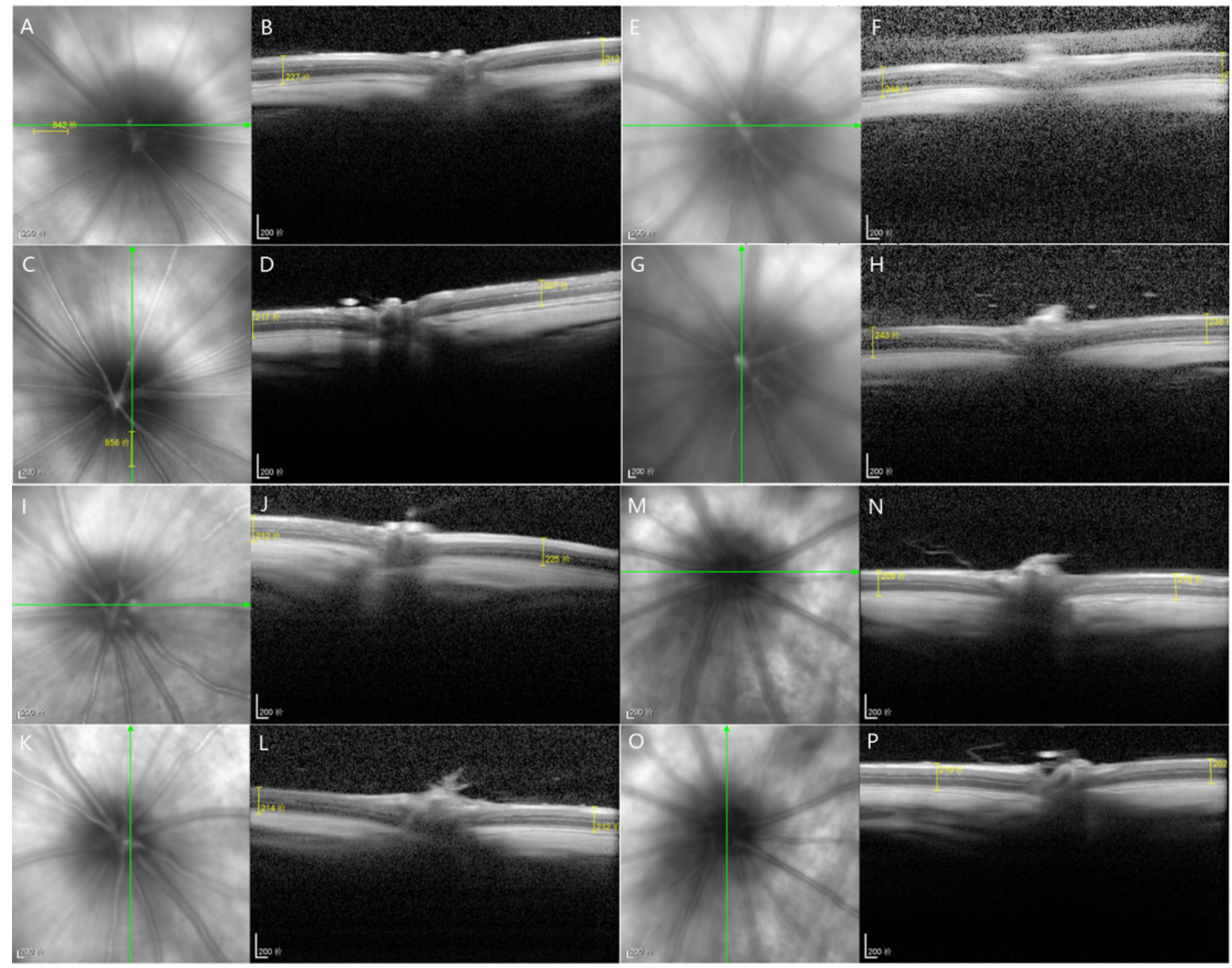


Figure 5

HE staining image of retina $* 400$ in each group of rats.(A): Group I-NC, (B): Group II-DM, (C): Group III-DM+CW, (D): Group IV-DM+Gli

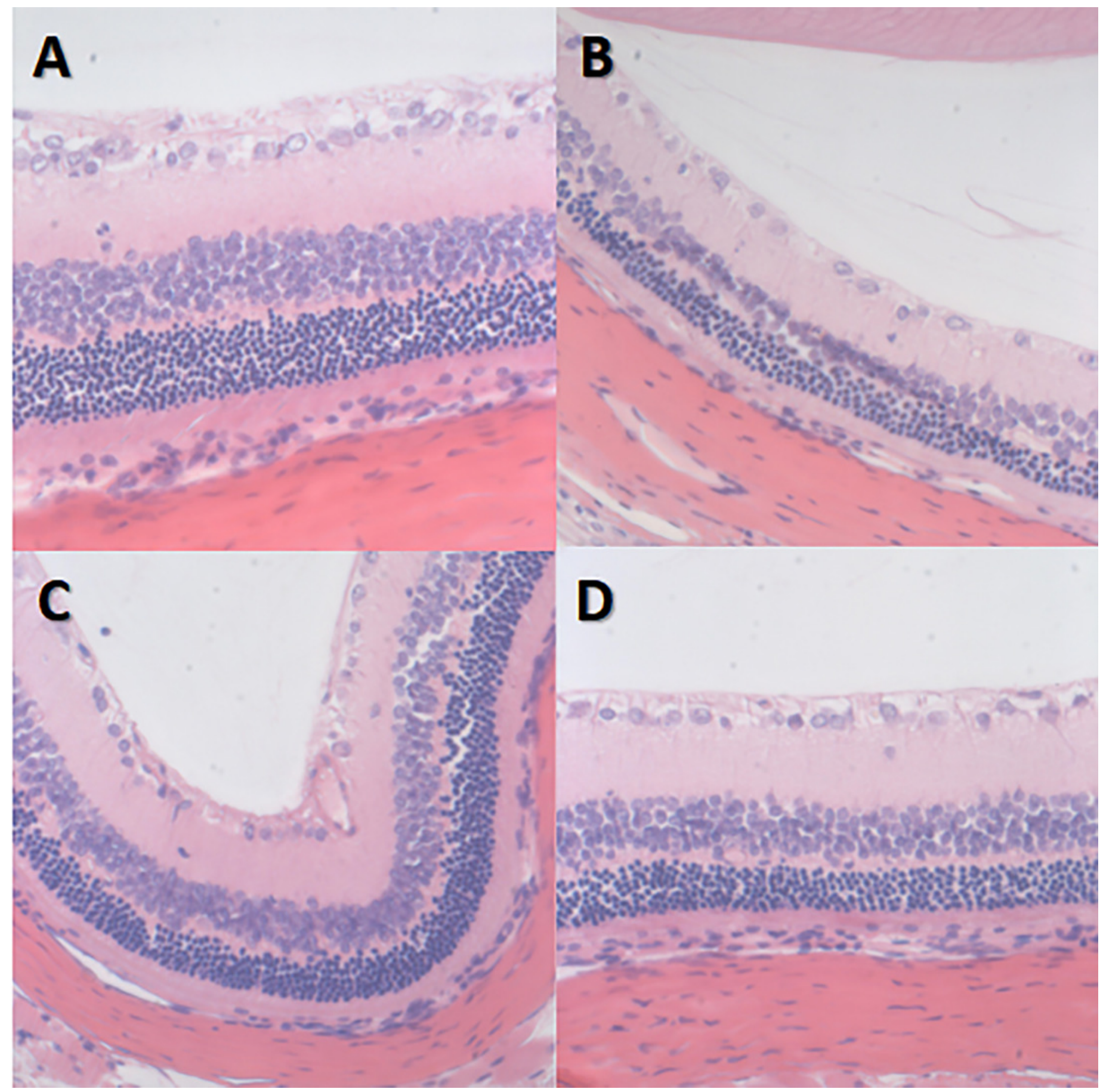




\section{Figure 6}

VEGF immunohistochemical staining of retinal sections $\times 600$ in each group of rats. $(A)$ : Group I-NC, (B): Group II-DM, (C): Group III-DM + CW, (D): Group IV-DM + Gli. Blue arrows indicate intraretinal granular layers, and yellow arrows indicate extraretinal

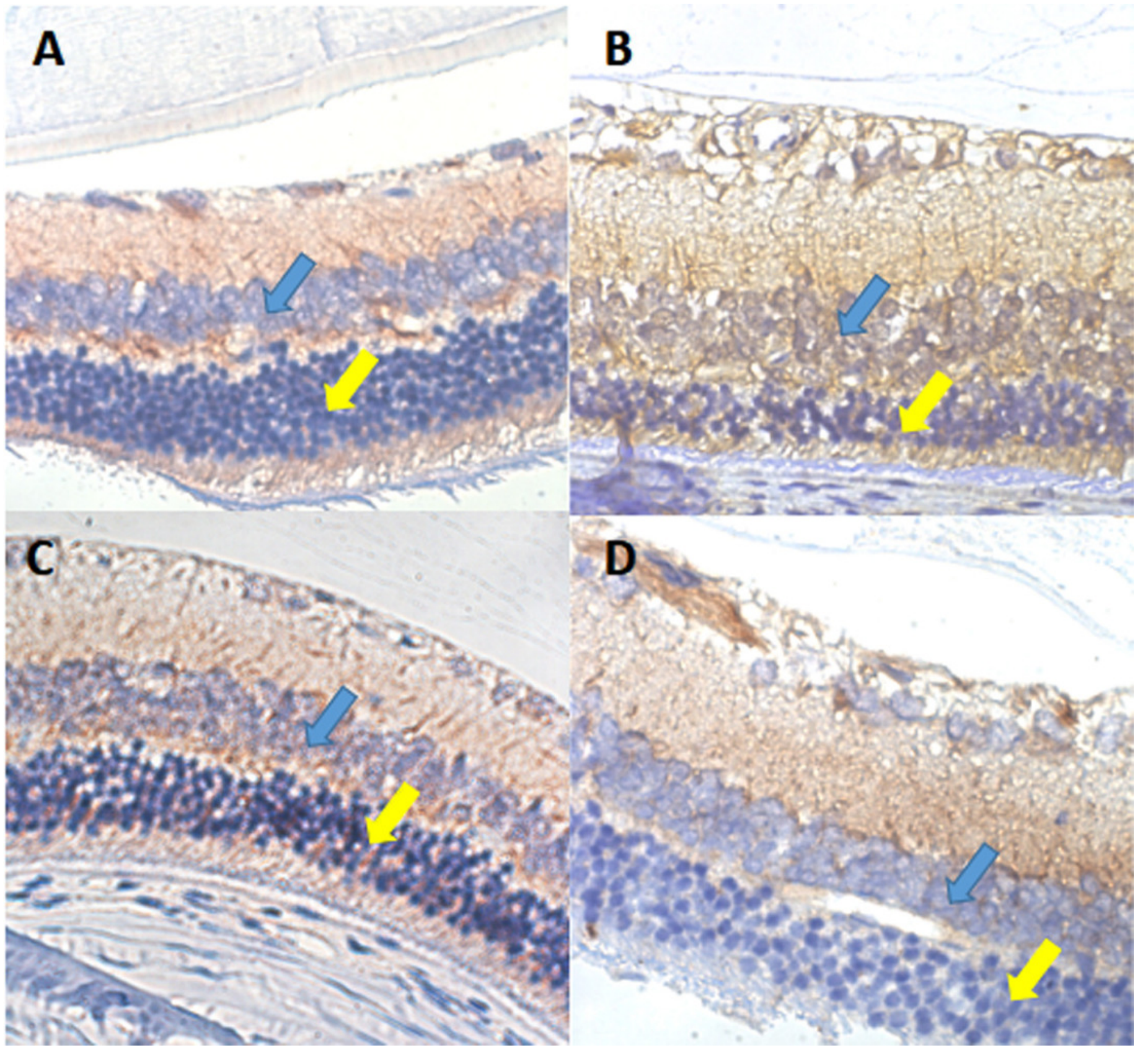




\section{Table $\mathbf{1}$ (on next page)}

Comparison of mean total retinal thickness at different time points in rats of each group 


$$
\begin{array}{ccc} 
& \text { Group III- } & \text { Group } \\
\text { Group I-NC Group II-DM } & \text { DM+CW } & \text { IV-DM+Gli }
\end{array}
$$

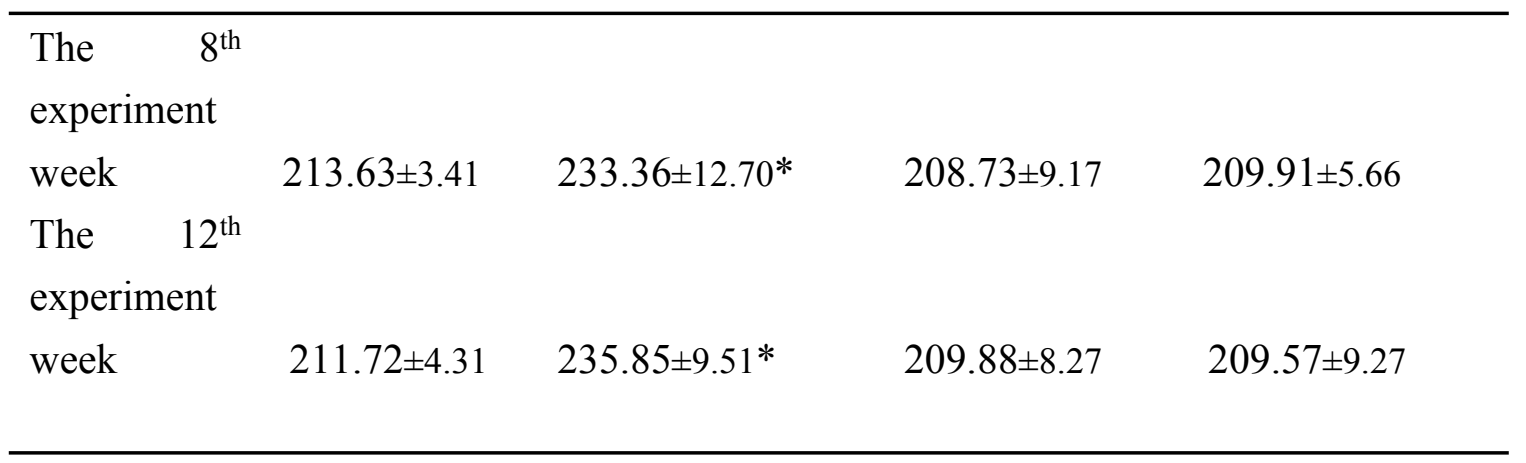

$2 * P<0.05$, Compared with Group I-NC

$3{ }^{\Delta} P<0.05$, Comparison with Group II-DM

4 